\title{
Malaria Control Efforts in the Era of COVID-19: A Call to Remain Vigilant in Endemic Areas
}

\author{
Eliningaya J Kweka ${ }^{1,2 *}$ (D) \\ ${ }^{1}$ Department of Medical Parasitology and Entomology, Catholic University of Health and Allied Sciences, \\ Tanzania \\ ${ }^{2}$ Division of Livestock and Human Diseases Vector Control, Tropical Pesticides Research Institute, Tanzania
}

*Corresponding author: Eliningaya J Kweka, Department of Medical Parasitology and Entomology, Catholic University of Health and Allied Sciences, Mwanza, Tanzania; Division of Livestock and Human Diseases Vector Control, Tropical Pesticides Research Institute, Arusha, Tanzania

\begin{abstract}
Success in controlling malaria has been reported across the world mainly through the use of long-lasting insecticidal nets, indoor residual spray, and appropriate diagnosis and treatment. However, in the era of COVID-19, more attention has been shifted to cases and mortality caused by the SARS-CoV-2 virus, which are jeopardizing the continuity of efforts attained so far in malaria control. This commentary proposes alternatives to be adopted during the COVID-19 pandemic to maintain the attained malaria control success.
\end{abstract}

\section{Keywords}

Pandemic, Malaria, Intervention, Lockdown, Endemic, COVID-19, SARS-CoV-2

\section{Abbreviations}

COVID-19: Coronavirus Disease 2019; IRS: Indoor Residual Spray; LLINS: Long Lasting Insecticidal Nets; WHO: World Health Organisation

\section{Background}

Malaria remains a major disease of public health importance in tropical and sub-tropical regions [1]. Global efforts in the control of malaria vector and parasites for the past two decades have shown a great decline in cases and related mortality [1]. Malaria vector control has been achieved through indoor residual spraying (IRS), long-lasting insecticidal nets (LLINs), and in some areas larval source management [1]. The high coverage of IRS and LLINs has caused a global decline of malaria vectors and cases of the disease from 2.7 Million in 2000 [2] to 450,000 deaths per year in 2019 [1]. Regardless of the vector populations decline due to intensive interventions implementation, the insecticide resistance increase and changes in feeding and resting behavior have enhanced low vector population sustained residual malaria transmission regions [1].

The current global pandemic caused by COVID-19 has shifted the attention of many malaria endemic and non-endemic countries to focus on the exponential increasing infections and mortalities caused by COVID-19. The trend shows that, from January to May, 2020 COVID-19 has killed 345,059 from January to date while malaria has killed 256,795 [3]. Currently, all countries in the WHO African regions have reported COVID-19 cases with 10 countries confirmed local transmissions [4,5]. At the moment, the COVID-19 recovery rates among infected patients are still low in most infected areas and more cases are reported on daily basis across the world both imported and locally transmitted [5]. At this time of pandemics, all governments in malaria endemic countries are putting lots of efforts and preparing to curb COVID-19 outbreaks, with minimal focus on vector borne disease control.

The WHO has warned the countries to be vigilant and increase a number of control effort against COVID-19 including frequent hand washing, social distancing and avoiding unnecessary social gathering [6]. At the present, there are reports of affecting the main supply chain of essential malaria control tools (such as long-lasting insecticidal nets, rapid diagnostic tests and antimalari-

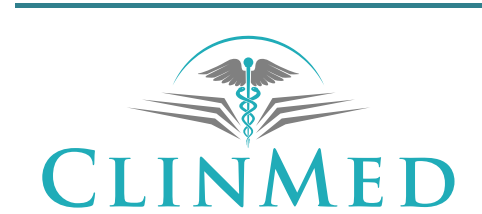

INTERNATIONAL LIBRARY
Citation: Kweka EJ (2020) Malaria Control Efforts in the Era of COVID-19: A Call to Remain Vigilant in Endemic Areas. Havana 2020. Int J Trop Dis 3:033. doi.org/10.23937/2643-461X/1710033

Accepted: June 17, 2020; Published: June 19, 2020

Copyright: (C) 2020 Kweka EJ. This is an open-access article distributed under the terms of the Creative Commons Attribution License, which permits unrestricted use, distribution, and reproduction in any medium, provided the original author and source are credited. 
al medicines) in several countries due to the lockdowns and from a suspension of importation and exportation of goods due to worries of COVID-19 spread [6].

The measures taken so far to reduce the spread of the virus include quarantine of suspects, family quarantine in reducing unnecessary movements, increasing quality of hygiene and report of all cases for proper management in the infected countries [4]. Meanwhile the families are at home, public health education given in all media should also emphasize the use of vector control including LLINs, and report of any malaria related case for diagnosis and appropriate treatment. On the other hand, government appropriate institutes should be responsible for disseminating information to the community on the necessity of keeping an eye on malaria disease operations.

This commentary suggests the following ways to be incorporated in COVID-19 cases management i) Presumptive malaria treatment of all suspected cases in community without the benefit of the diagnostic tools results because of limited of many services in malaria endemic areas, and ii) Provision of mass drug administration in communities as the diagnosis of cases and follow-up may not be possible. This method will benefit a wide coverage based on difficulties of people gathering for mass screening and treatment in worries of COVID-19 spread. These two major suggestions can be useful as they will reduce the mortality of patients and keep the health workers safe from COVID-19 infections. The major efforts which were directed to the control of malaria parasites and vector should be maintained to eliminate pools and residual malaria transmission hotspots. Assessment of insecticides and antimalarial drug sensitivities should be continuous at this time.

National malaria control program across malaria endemic areas should be facilitated with external and internal funds to enhance rapid response to any malaria cases reported in collaboration with health system structure. The national and local program centered in malaria control should continue to avail the core preventive and case management interventions even at this great risk of transmission of COVID-19 in malaria endemic countries.

There is a great worry that, there can be suspension of all activities including LLINs distribution, IRS progress and abatement due to the risk of workers and community members to contract COVID-19. The WHO is coordinating malaria control efforts through three stages; linking countries, regions and WHO headquarter. All that is to ensure no chain break in coordination of malaria control tools supplies for highly malaria endemic countries and ensure that the efforts access to malaria treatment, diagnosis and prevention services. Of limiting the spread of COVID-19 should not hinder

\section{Going Forward}

The national malaria control programmes in malaria endemic areas of African region should consider a number of activities during this time to sustain attained results; i) Initiate malaria responsive team for any reported cases for treatment and management country-wide, ii) Maintenance of door to door LLINs distributions, iii) Community members should be sensitized to be proactive to report all emerging malaria related cases to community-based health workers to take care off, iv) The provision of mass drug administration should be considered with care to communities to avoid spread of COVID-19 and protection of health workers when deemed necessary, v) The malaria control team members in all endemic countries should also be incorporated in national COVID-19 management team so as to ensure maximum protection and collection of emerging cases. This will enable collection of data for both COVID-19 and malaria related cases with the same personnel and at minimal risk of infection, and vi) During cases collection, the health workers should follow all measures for the protection and prevention of COVID-19 spread.

\section{Conclusion}

Although, COVID-19 and mortality related cases are expanding exponentially, community should remain vigilant and implement WHO recommended safe keeping practices from corona virus while maintaining safely malaria control tools such as provision of LLINS and anti-malaria drugs programmes.

\section{Declarations}

\section{Ethics approval and consent to participate}

Not applicable.

\section{Consent for publication}

Not applicable.

\section{Availability of data and materials}

Not applicable.

\section{Funding}

There was no financial matter to be declared.

\section{Authors' contributions}

EJK conceived and wrote the commentary to submission.

\section{Acknowledgements}

I would like to appreciate the help I was given by Lucy Kisima (TPRI Librarian) for literature access. Dr Stephen Munga from KEMRI-Kisian is appreciated for his comments in first draft.

\section{References}

1. (2019) World malaria report 2019. World Health Organisation, Geneva. 
2. (2000) WHO expert committee on malaria: Twentieth report. World Health Organization, Geneva.

3. (2020) Global Deaths Due to Various Causes and COVID-19 in 2020.
4. (2020) Coronavirus disease 2019 (COVID-19): Situation reports. WHO, Geneva.

5. (2020) COVID-19 distribution globally.

6. (2020) Malaria and the COVID-19 pandemic. 